\title{
A Novel Method to Predict the Well Inflow Performance Relationships Curves By Artificial Intelligence Techniques
}

\author{
Pedram Farmahini Farahani ${ }^{1}$, Ali Esfandiari Bayat ${ }^{2 *}$, Roozbeh Rafati $^{1}$ \\ ${ }^{1}$ Department of Petroleum Engineering, Science and Research Branch, Islamic Azad University,
}

Tehran, Iran

${ }^{2}$ Department of Reservoir Engineering, Faculty of Petroleum and Chemical Engineering, Science and Research Branch, Islamic Azad University, Tehran, Iran

*Corresponding Author: Ali Esfandiari Bayat, Department of Reservoir Engineering, Faculty of Petroleum and Chemical Engineering, Science and Research Branch, Islamic Azad University, Tehran, Iran

\begin{abstract}
In flow performance relationships (IPRs) represent the relation between well flow and the bottom hole pressure; the performance prediction of IPRs plays a fundamental role in the optimization of production operations and subsequently to design an artificial dislocation to select the most appropriate production scenario. The purpose of this study is to consider the effect of several important parameters on IPR curves by the utilization of a multilayered perceptron artificial neural network. The inputs of this system are the well pressure, the average reservoir pressure, and the maximum flow rate, while the output of this system was assumed pumped oil. Since then by using an artificial neural network code with MATLAB software and coupling it with the genetic codes, a random search algorithm to predict the IPR curves are plotted. Consequently, according to the results of this study, the average reservoir pressure has a profound effect on the IPR curves and the genetic algorithm plays an essential role in determining the weights, bias, and the structure of the artificial neural network. in respect of the way, four percent error in actual data from the network in the test data section indicates that this algorithm is based on a random search can well identify the relationship between inputs and outputs. Furthermore, artificial neural networks can be used with good precision and credibility rather than a reservoir simulator to generate other data due to the acceptable results of data matching.
\end{abstract}

Keywords: Inflow performance relationship curves, Artificial neural network, Average reservoir pressure, Genetic algorithm

\section{INTRODUCTION}

The appropriate prediction of IPR curves is considered as a significant influence in determining the reservoir performance. Thereby, determination of pressure drop value, flow rate measurements into the reservoir and should be taken into the consideration in the reservoir optimization. Artificial neural networks have been widely developed in the coming decades by the administration and implying the structure of neural networks according to the implantation of life body by the purpose of modeling the reservoir characteristics by these artificial networks. The use of artificial neural network is a new technique in the oil industry. These networks can be used to identify patterns, data gatherings, recover the discrepancies, and filtering the occurred noises. Artificial neural network design is a simple and accurate way to estimate important reservoir characteristics, including porosity, from well charts data. Shippen et.al (2001) applied a neural network model to predict fluid holdup in two phase flow in the horizontal direction, and subsequently, the proposed analytical modeling demonstrated the better performances in this issue [1]. Zambrano (2002) used a neural network model to predict dew point pressure for retrograde gases which is illustrated that the provided model was better estimation techniques for the existed correlations [2]. Incompatible results state again by Camacho and Raghavan; it was noted that the predicted IPR by Vogel indicates the definition of flow efficiency should also represent quadratic forms of the depression equation rather than the definition of the righthand side of the reflection [3-5]. Furthermore, they concentrated on the utilization of a numerical simulation to investigate the effect of the skin on the well production by solution gas drive. Subsequently, they showed that the effect of the delineation curve on the skin effects does not change the quadratic equation in a linear state [6]. Clins (2004) analyzed the results of a non-IPR curve and 
concluded that the curve of the IPR was affected by the effect of the skin. The proposed model investigated the factors of affecting the dimensionless IPR function and hence, the bubble pressure point and pressure of reservoir drainage had a significant influence on the differentiations of IPR curves [7]. Thereby, the purpose of this study was to determine the performance of IPR curves and optimize this graph using genetic algorithm network [8-15].

\section{Materials AND Methods}

The simulation carried out in this paper was based on the studies by Huppert et.al (1982) and the data which was used to propose the model was extracted from the Weinstein et.al (1986) [16]. Furthermore, IPR curves were simulated in the Black oil model in Eclipse software to provide an analytical comparison with the artificial neural network techniques in which the utilization of genetics algorithm should be trained to the system. It should be noted that the IPR curves were generally plotted the bottom hole pressure versus flow rate at a stable reservoir pressure or used as a specific recycling rate. In this study, the average reservoir pressure was used in the simulation processes to identify the well operation. To do this, different reservoir pressure values $(3000,2500,2000,1500$ psia) were considered which is relatively close to the initial reservoir pressure of 3600 psia. On the other hand, the purpose of IPR curves is to detect the relationship between the well flow pressure (Pwf) and the flow rate(Qo), hence, the set of statistic wells with the pressures of 14.7, 250, 500, 750, 1000,1500 psia are taken into the consideration. Figures 1 and 2 show an example of how to find the time in the FPR curves and its relationship with the FOPR curves.

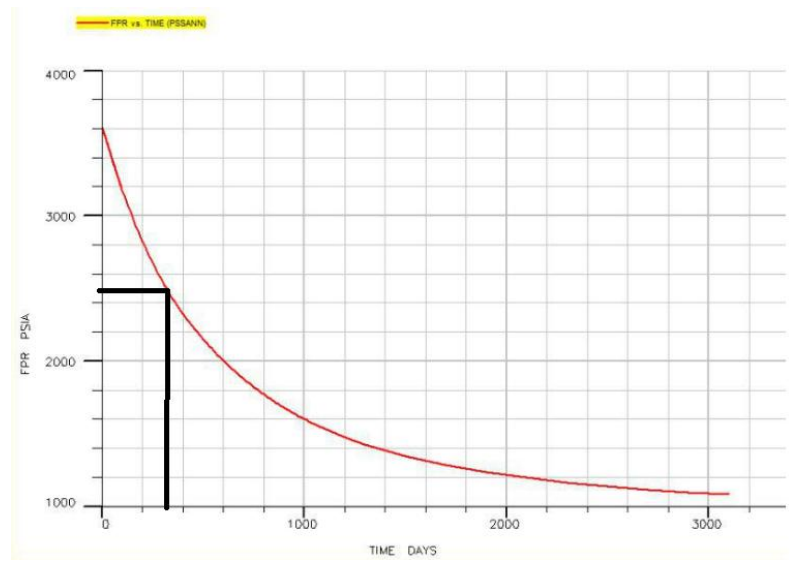

Figure1. Calculated pressure at the pressure of 2500 psia for the studied reservoir

As it is shown in Figure 1, at an average reservoir pressure of 2500 psia, the FPR value is considered equally as the average reservoir pressure and in the next stages the time is observed and recorded. To be more accurate and virtually eliminate the occurred errors, Eclipse software are being used to control the discrepancies. It should be noted that in the most of the 24 survey modes, there was a need for a double-checking because by minimizing the steps of the program's performances, it was not possible to fit the corresponding numbers into specific time.

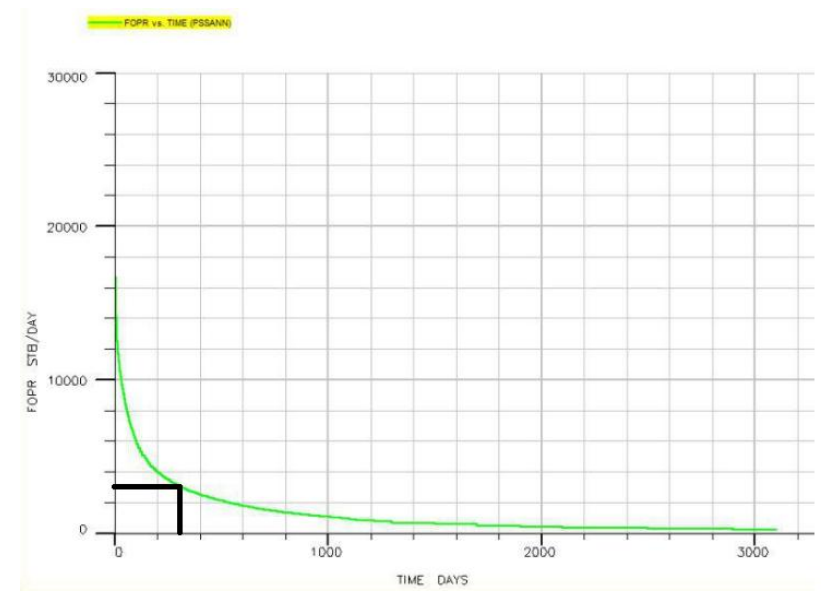

Figure2. The relationship between FPR and FOPR at the pressure of 2500 psia for the studied reservoir 
By determining the time to reach the field pressure to the average reservoir pressure, the corresponding discharge at the measurement time was obtained. Figure 2 illustrates an example of 24 different modes (different flow conditions and compression conditions) of the implementation of the Eclipse. As indicated by the black lines, the FOPR output can produce the amount of daily flow from the field. It should be noted that for this purpose, the report of the Eclipse is required. In Figures 3-6, a set of IPR curves were obtained according to the above method. The above set of points for different values of reservoir pressure and bottom hole pressures can be used for training and testing of artificial neural networks and thus the accuracy of artificial neural network for reproduction of different data is investigated.

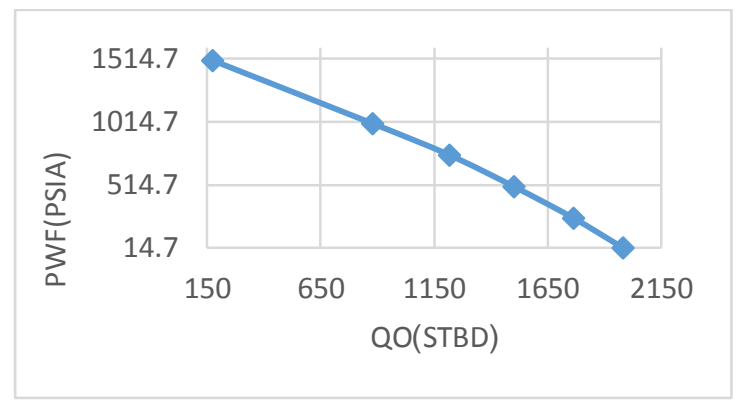

Figure3. IPR curves at the pressure of 1500 psia for the studied reservoir

Figure 3 shows how the IPR curve changes for an average reservoir pressure of 1500 psia. As it is evident, we were able to plot the IPR curve by reading 5 points for each reservoir average pressure. However, increasing the number of points and checking a higher state leads to a rise in the accuracy of the curvature of the concavity in terms of mathematical rules. It should be noted that the straight line of the curve in Figures 3 and 4 depicted that the production of the reservoir in these conditions is made over the bubble point pressure.

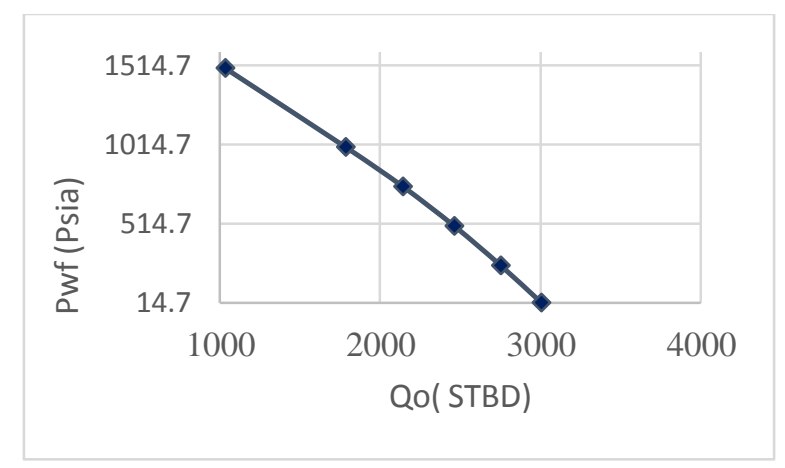

Figure4. IPR curves at the pressure of 2000 psia for the studied reservoir

Figure 4 illustrates the relation between the flow behavior for the average reservoir pressure of 2000 psia. The apparent difference between Figure 4 and Figure 3 is the value of AOF. As a result, regarding the increase in the average reservoir pressure, the amount of produced gas is also increased, and one of the things that can be found is that the production rate is at the pressure of 14.7 psia.

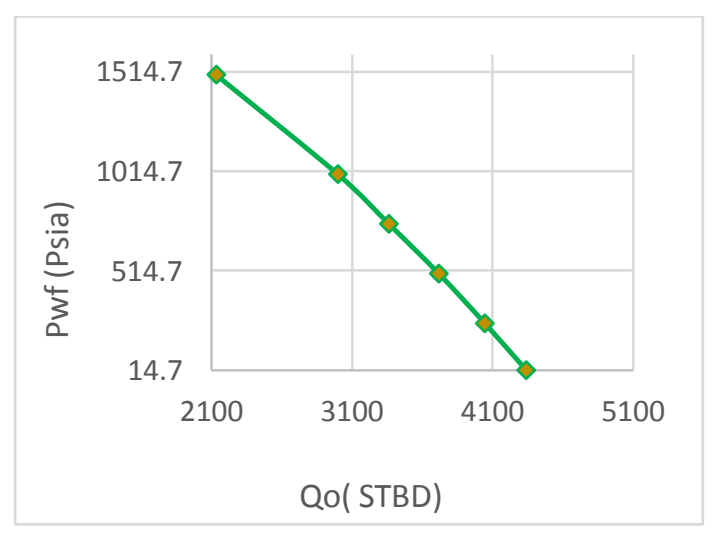

Figure5. IPR curves at the pressure of 2500 psia for the studied reservoir 
Figure 5 shows the IPR curve for an average reservoir pressure of 2500 psia. As can be seen from Figure 5, the average reservoir pressure increase of 500 units (compared to the previous state, with the 2000 psia average reservoir pressure) has increased the AOF by 1300 barrel per day. Therefore, the average reservoir pressure will play a significant role in the alterations of IPR curves.

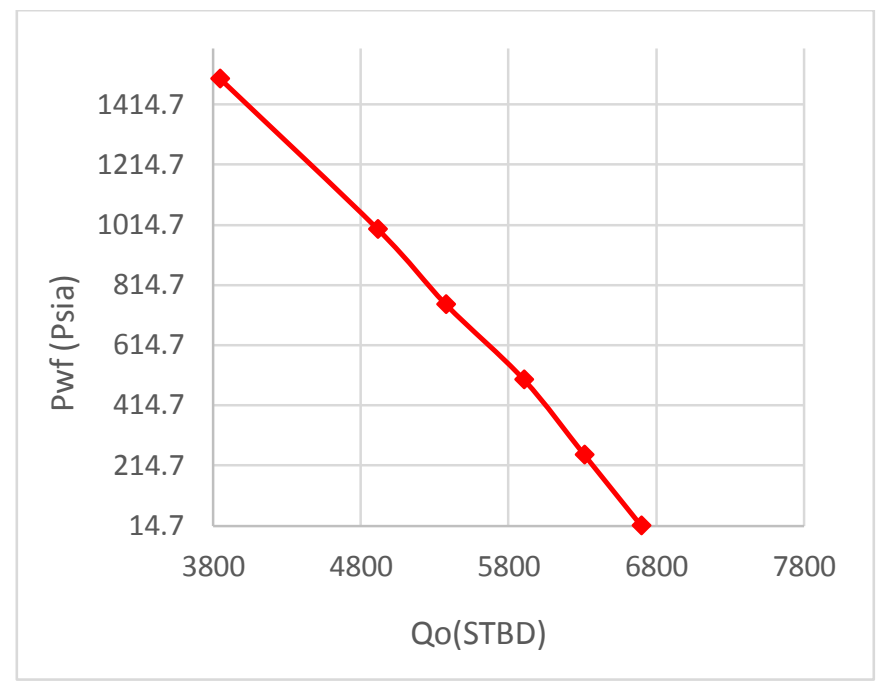

Figure6. IPR curves at the pressure of 3000 psia for the studied reservoir

As it is clarified in Figures 3-6, the average reservoir pressure is higher than the bubble pressure, the gas was not released from the fluid, however, regarding the gas release, the slopes of the curves are increased. Moreover, as it is clear, for different average reservoir pressures, the gradient and constituent points of IPR curves are different. In general, the well productivity has decreased gradually with the increase of reservoir drainage. The reason for this is the decrease of reservoir pressure and the gas saturation rise, which caused to increase the oil flow resistance. In this study, by the simulation analysis with Eclipse Software in the pressure of 14.7 psia, the flow rate is being calculated by the absolute open flow rate. For a more comprehensive review of the relationship between inputs data (average reservoir pressure, well pressure and absolute open flow) on the target output (oil flow rate), the artificial neural network of multilayered perceptron is trained by the genetic algorithm. The related data which is used in the artificial neural network are summarized in Table 1.

Table1. Artificial neural network parameters in the optimization procedures

\begin{tabular}{|c|c|}
\hline Population Size & 300 \\
\hline Generations & 300 \\
\hline Selectivity Function & Stochastic Uniform \\
\hline Intersection Function & Arithmetic \\
\hline Intersection Percent & $70 \%$ \\
\hline Mutation Function & Discrete \\
\hline Mutation Value & 0.075 \\
\hline
\end{tabular}

\section{RESULTS AND DISCUSSION}

\subsection{Artificial Neural Network Predictions}

By the Utilization of 24 datasets which is obtained from the implementation of the Eclipse simulation model in different conditions and applied the toolbox artificial neural network in Matlab software by a little change in the written codes, the perceptron artificial neural network structure of the multi-layers is being analyzed. In respect of the way, the genetics algorithm is considered as the optimization algorithm. To do this, the number of hidden network layers was assumed to be between 2 and 3 variable layers, and the neurons of each of the layers changed from 3 to 8 . Therefore, to achieve the optimum oil flow rate function in the neural network, the conditions for the improvement of the network structure were implemented to the system and subsequently an optimum network was selected which is contained the lowest RMSE for the dataset. Moreover, the condition for reaching the end of the network was assumed the certain number of courses (100 courses). The related information on the selection of neural networks is statistically explained in Table 2. 
A Novel Method to Predict the Well Inflow Performance Relationships Curves By Artificial Intelligence Techniques

Table2. Required data and optimal conditions for the artificial neural network of oil production in the studied reservoir

\begin{tabular}{|c|c|}
\hline Network & Cumulative oil production \\
\hline Number of neurons in the input layer & 3 \\
\hline Number of hidden layers & 2 \\
\hline Number of neurons in hidden layers & {$[4$ 4] } \\
\hline Activation Functions & [Tanh Tanh Linear] \\
\hline Training Algorithm & Genetic Algorithm \\
\hline Number of neurons in the output layer & 1 \\
\hline Number of training data & 5 \\
\hline Number of Test Data & 19 \\
\hline
\end{tabular}

\subsection{Oil Production Rate Network}

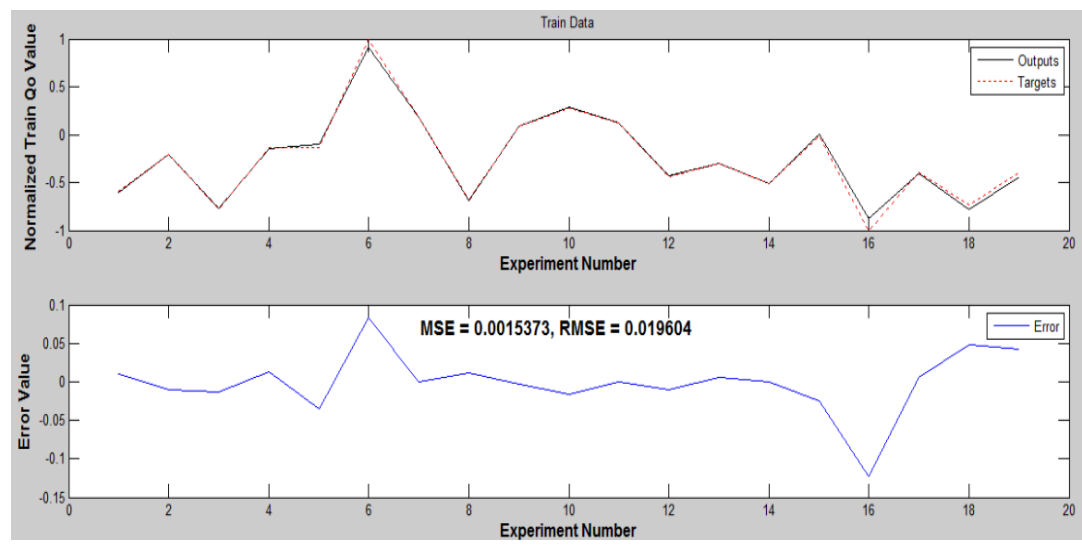

Figure7. Training normalized data for the target and output data of cumulative oil production (scenario\#1)

According to the Figure 7, the actual data (derived from the implementation of the Eclipse model) and the result of an artificial neural network for training data are depicted and for the total number of training data which is 19 , the simulator results represent a $2 \%$ acceptable error for the data in the training section. It should be noted that the normalized data (between -1 and 1) is used to eliminate the effect of different data scales. In addition, it is worth noting that the data are randomly selected for the training and testing section of the network to test the accuracy and reliability of the network which is caused to reduce the probability of data overtraining.

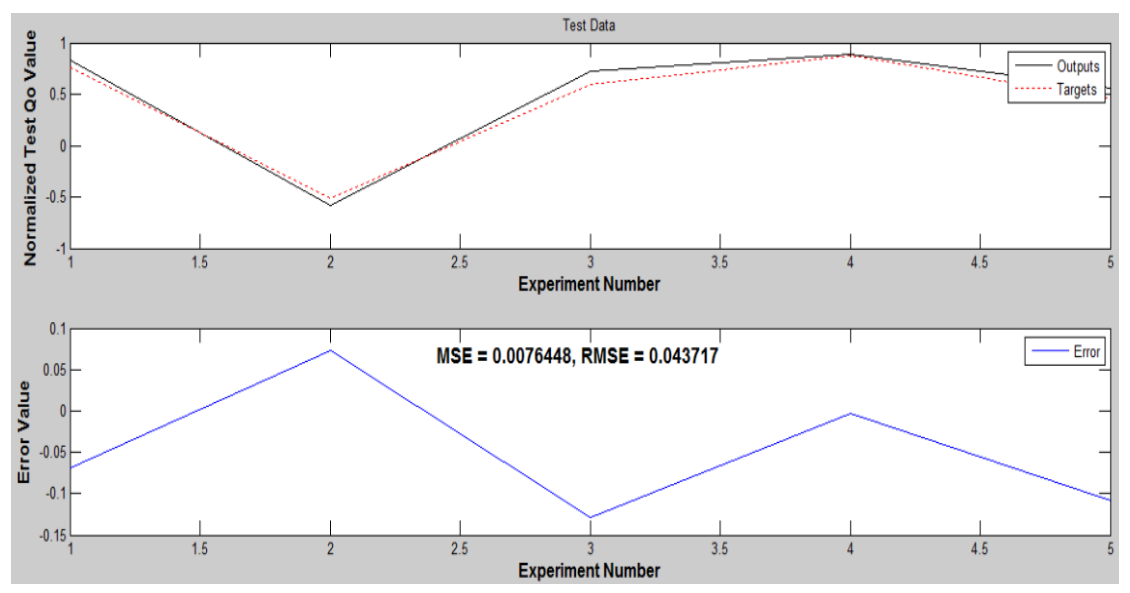

Figure8. Training normalized data for the target and output data of cumulative oil production (scenario\#2)

Figure 8 shows that the artificial neural network, in which input data was used randomly for training and testing data, has been able to predict test data in a proper way. Although the number of data related to network training was low (19 trials), the results of the RMSE error of the test data is about $4 \%$ which indicates that the network has been able to predict the flow data and output behavior of the network to make a negligible error offer. Furthermore, the network in spite of having a limited number of data in the test section ( 5 random data), it is correctly identified the relationship between different parameters. Therefore, it would be able to perform appropriately for different input values. 


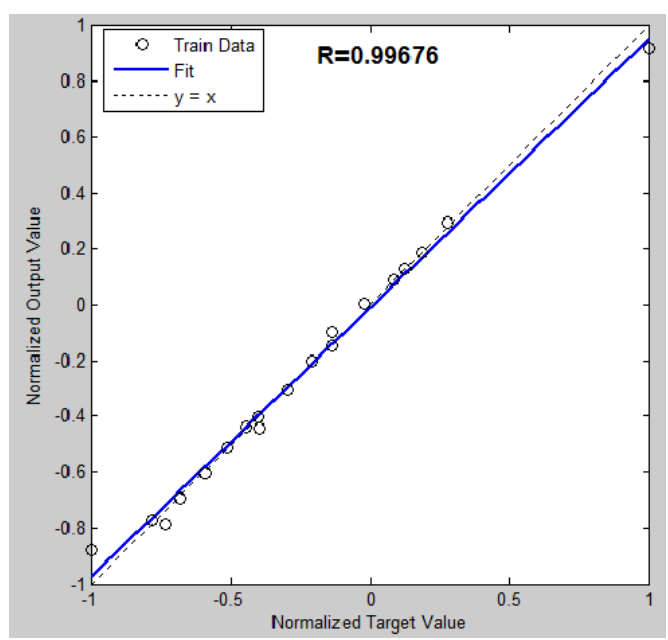

Figure9. Regression Curves for the target data (by Eclipse software) and output data (neural network)$R=0.99676$

As it is schematically demonstrated in Figure 9, the linear regression between the target data (derived from the simulation of the black oil model in Eclipse) and the output data (derived from the perceptron artificial neural network of the multilayer perceptron) was plotted. The more adaptation of the data with each other are depicted the reliability of the proposed model with the results of neural network. In addition, the regression coefficient for this correlation number is about 100 percent, which indicates that the relationship between actual data and network output for highly linear training data is proper and the network has been able to perform adequately.

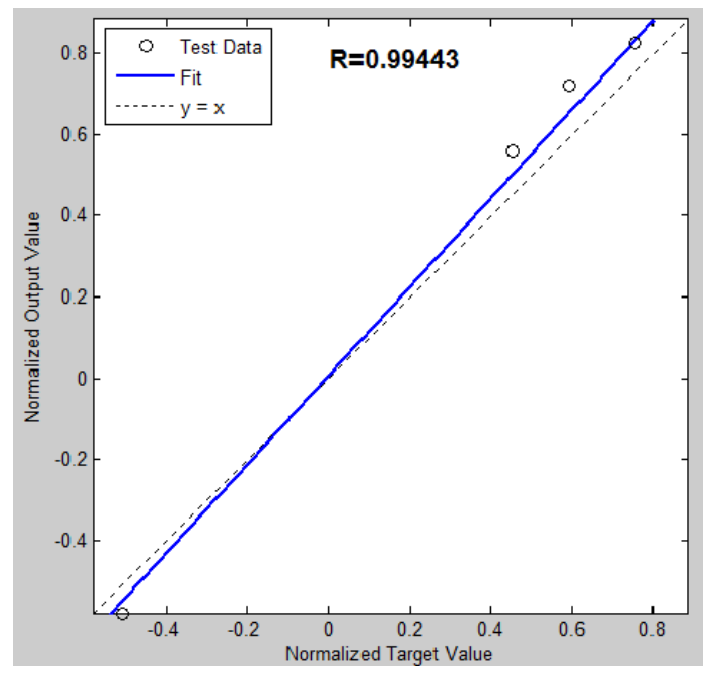

Figure10. Regression Curves for the target data (by Eclipse software) and output data (neural network)$R=0.99443$

As it is evident in Figure 10, there is a linear correlation between actual data and network output for cumulative flow test data as same as figure 9. Moreover, the regression coefficient is about $99 \%$, which is a proper relation with the outputs of the test data and regarding the low number of provided available data has been successfully applicable in predicting the IPR curves. This precise accuracy of the randomized test data indicates that the artificial neural network is able to be used instead of the reservoir simulator. Therefore, in general, the multi-layered perceptron artificial neural network would be successfully implemented by the genetic algorithm.

\section{CONCLUSION}

This study clarify that the genetic algorithm plays a fundamental and precise role in regulating weights, biases, number of hidden layers and neurons of each layer. Hence, it significantly reduces the error of the training and root data in this precision. A significant error of $2 \%$ in training data and $4 \%$ in test data is evidence of this accuracy. Furthermore, the following results are the major conclusions of this comprehensive study; 
- The accuracy of about $100 \%$ in regression curves indicates the reliability of the neural network in other performances.

- The utilization of a reservoir simulator in other scenarios is unnecessary. The successful performance of artificial neural network in the estimation of (daily production flow) eliminates the need of using the Eclipse software to produce IPR curves. As a result, we can predict IPR curves only by relying on artificial neural networks.

- An artificial neural network made using the genetic algorithm for training which was able to evaluate the relationship between inputs (bottom pressure, reservoir pressure, and maximum flow rate) and output (oil production) accurately.

- Learning and testing will be used with proper confidence for other data.

- The average reservoir pressure is a key parameter and influences the process of IPR curves. Because the flow correlations represent the prediction of how to change the pressure of the well in terms of the production rate in which the average reservoir pressure is known.

\section{REFERENCES}

[1] M. E. Shippen, "Development of a Neural Network Model for the Prediction of Liquid Holdup in TwoPhase Horizontal Flow," Texas A \& M University, 2001.

[2] A. Gonzalez Zambrano, "Development of neural network models for the prediction of dewpoint pressure of retrograde gases and saturated oil viscosity of black oil systems," Texas A\&M University, 2002.

[3] J. V Vogel and others, "Inflow performance relationships for solution-gas drive wells," J. Pet. Technol., vol. 20, no. 1, pp. 83-92, 1968.

[4] M. B. Standing, "Inflow Performance Relationships for Damaged Wells Producing by Solution Gas Drive Reservoirs," J. Pet. Tech., pp. 1399-1400, 1970.

[5] M. B. Standing, "Concerning the calculation of inflow performance of wells producing from solution gas reservoirs," JPT, pp. 1141-1157, 1971.

[6] R. G. Camacho, R. Raghavan, and others, "Inflow performance relationships for solution-gas-drive reservoirs," in SPE Production Operations Symposium, 1987.

[7] M. A. Klins, M. W. Majcher, and others, "Inflow performance relationships for damaged or improved wells producing under solution-gas drive," J. Pet. Technol., vol. 44, no. 12, pp. 1-357, 1992.

[8] Saputelli, L., Malki, H., Canelon, J. and Nikolaou, M.: "A Critical Overview of Artificial Neural Network Applications in the Context of Continuous Oil Field Optimization," paper SPE 77703 presented at the 2002 SPE Annual Technical Conference and Exhibition, San Antonio, Texas, 29 September - 2 October.

[9] T.F. Bene, J.M. Benesch ." Optimized Big Bore Gas Wells for Qatar North Field.” Paper SPE 12458 presented at the 2008 ExxonMobil Production Company, ExxonMobil Development Company.

[10] Kharrat, R.; Razavi, S. M., (2008). "Determination of Reservoir Model from Well Test Data, using Artificial Neural Network", Scientia Iranica, Vol. 15, No. 4, pp 487-493.

[11] Tariq, Z., Abdulraheem, A., Khan, M. R., \& Sadeed, A. (2018, March). New Inflow Performance Relationship for a Horizontal Well in a Naturally Fractured Solution Gas Drive Reservoirs using Artificial Intelligence Technique. In Offshore Technology Conference Asia. Offshore Technology Conference.

[12] Rubio, E., Caicedo, S., BinAmro, A., \& Davila, R. (2017, May). Novel Well Modelling Approach Enhances Daily Production Rate Estimation and Allocation Process in an Intelligent Oil Field, Abu Dhabi. In SPE Intelligent Oil and Gas Symposium. Society of Petroleum Engineers.

[13] Bertone, E., O'Halloran, K., Stewart, R. A., \& de Oliveira, G. F. (2017). Medium-term storage volume prediction for optimum reservoir management: A hybrid data-driven approach. Journal of cleaner production, 154, 353-365. Allawi, M. F., Jaafar, O., Hamzah, F. M., Abdullah, S. M. S., \& El-shafie, A. (2018). Review on applications of artificial intelligence methods for dam and reservoir-hydro-environment models. Environmental Science and Pollution Research, 1-24.

[14] Soleimani, M. (2017). Well performance optimization for gas lift operation in a heterogeneous reservoir by fine zonation and different well type integration. Journal of Natural Gas Science and Engineering, 40, 277287.

[15] Shokri, A., Azdarpour, A., \& Honarvar, B. (2017). Analysis of inflow performance relationship and reservoir characteristics using Saphir software. An International Peer Reviewed Open Access Journal For Rapid Publication, 145.

[16] Weinstein, H. G., Chappelear, J. E., \& Nolen, J. S. (1986, March 1). Second Comparative Solution Project: A Three-Phase Coning Study. Society of Petroleum Engineers. doi:10.2118/10489-PA 


\section{AUTHORS' BIOGRAPHY}

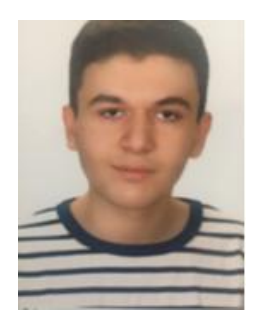

Pedram Farmahini Farahani, Master degree of Petroleum Engineering at Islamic Azad University, Tehran science and research Branch. My current interests in petroleum engineering sciences include a full-field study of Data Gathering, Reservoir Simulation, History Matching and Prediction.

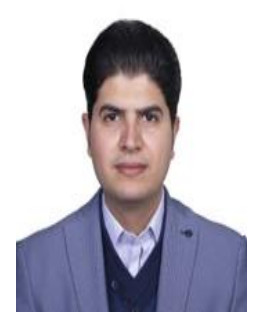

Dr. Ali Esfandiari Bayat, Post Doctorate Degree of Petroleum Engineering and he is assistant professor and M.SC. and doctoral student supervisor at Department of Reservoir Engineering, Faculty of Petroleum and Chemical Engineering, Science and Research Branch, Islamic Azad University, Tehran, Iran

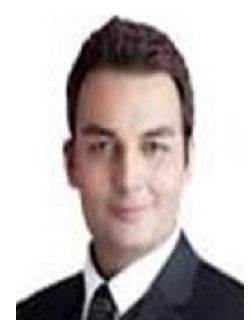

Dr. Roozbeh Rafati, is assistant professor and M.SC. and doctoral student supervisor, at Islamic Azad University, Tehran science and research Branch, where he specializes in reservoir engineering and operates such related projects in the university.

Citation: Pedram Farmahini Farahani, et.al. (2018). “A Novel Method to Predict the Well Inflow Performance Relationships Curves By Artificial Intelligence Techniques", International Journal of Petroleum and Petrochemical Engineering (IJPPE), 4(3), pp.13-20, DOI: http://dx.doi.org/10.20431/2454-7980.0403002

Copyright: (C) 2018 Authors. This is an open-access article distributed under the terms of the Creative Commons Attribution License, which permits unrestricted use, distribution, and reproduction in any medium, provided the original author and source are credited 